\title{
Identification of Serum Biomarkers and Pathways of Systemic Lupus Erythematosus with Skin Involvement Through GC/MS-Based Metabolomics Analysis
}

\section{Yongyi Xie* \\ Baoyi Liu* \\ Zhouwei Wu}

Department of Dermatology, Shanghai General Hospital, Shanghai Jiao Tong University School of Medicine, Shanghai, 200080, People's Republic of China

*These authors contributed equally to this work
Correspondence: Zhouwei Wu Department of Dermatology, Shanghai General Hospital, Shanghai Jiao Tong University School of Medicine, Shanghai, 200080, People's Republic of China Email 418950049@qq.com
Purpose: Skin involvement is the second most common symptom of systemic lupus erythematosus (SLE), and the prevention of skin lesion development might benefit to lessen the system inflammation burden in SLE. However, the mechanisms of skin lesion in SLE remain unclear.

Patients and Methods: Metabolome based on gas chromatography-mass spectrometry (GC-MS) was used for comparison of serum metabolism among 11 SLE patients with skin lesion (SL), 10 SLE patients without skin lesion (SNL), and 16 healthy controls (HC). The analysis of metabolism profiles was through LUG database, Human Metabolome Database (HMDB) as well as Kyoto Encyclopedia of Genes and Genomes (KEGG).

Results: A total of 14 most meaningful metabolites were found in SL patients compared to SNL patients, and 19 metabolic pathways were enriched. Meanwhile, L-alpha-aminobutyric acid, dehydroascorbic acid, glycine, and L-tyrosine achieved an area under receiveroperating characteristic (ROC) curve of $0.8636,0.8091,0.7727$, and 0.7636 , respectively, indicating their diagnostic potential for SL patients. In addition, the combined model of L-alpha-aminobutyric acid and dehydroascorbic acid provided better diagnostic accuracy.

Conclusion: The metabolomic features of SLE patients with skin lesion could be detected by GC/MS assay. Our study tried to provide new insights into the mechanism of SLE skin injury. Further validation of these findings through larger sample size studies may contribute to the use of metabolic profile analysis.

Keywords: metabolism, systemic lupus erythematosus, biomarker

\section{Introduction}

Systemic lupus erythematosus (SLE) is a complex and inflammatory autoimmune disease, resulting in multiple system damage. ${ }^{1}$ Followed by kidney, skin is the second most common organ affected in SLE patients. ${ }^{2}$ Approximately $72 \%$ to $85 \%$ of SLE patients have skin manifestation. ${ }^{3}$ It has been reported that nuclear antigen released by aberrant apoptotic keratinocytes in skin lesion could into the circulation, cause inflammatory response in different organs, and initiate the systemic disease flares. ${ }^{2,4}$ Our previous study also suggested that the severity of skin inflammation in cutaneous LE was associated with the progress of CLE to SLE. ${ }^{5}$ These data imply that the prevention of skin lesion development might benefit to lessen the system inflammation burden in SLE.

Several studies have tried to elucidate the mechanism of skin inflammatory development in SLE patients. Previous study found that injection of lupus serum 
could cause skin inflammation in wide-type mice, which could not be observed by IgG-depleted lupus serum, ${ }^{6}$ indicating that $\operatorname{IgG}$ might be involved in the skin lesion development in SLE. Meanwhile, abnormal plasmacytoid dendritic cells were found to be accumulated in SLE skin lesion. Furthermore, cytokines such as TNF, interleukin-1 (IL-1), IL-12 also contributed to the development of skin lesion in SLE. ${ }^{2}$ In addition to aberrant autoantibodies, immune cells, and cytokines, the ratio of aryl hydrocarbon receptor (AhR) in $\mathrm{T}$ cells also have been reported to be associated with skin injury by regulating the balance of Th17 and Treg cells. ${ }^{7}$ These evidences suggest that the exact pathogenesis of skin lesion development in SLE are much more complicated than expected.

Recently, metabolic profiling which can detect and quantify low molecular weight molecules (metabolites) in biological fluids has great value in identifying biomarkers in different diseases, including SLE. Zhang et $\mathrm{al}^{8}$ have reported that serum L-pyroglutamic acid could act as a potential diagnostic biomarker to distinguish SLE patients from healthy people. Other studies demonstrated that dysregulated metabolic pathways, such as aminoacyl-tRNA biosynthesis, was observed in fecal samples of SLE patients. ${ }^{9,10}$ In addition, Kalantari et al $^{11}$ identified different metabolomics associated with lupus nephritis (LN), which could be used for non-invasive diagnosis of LN. Whereas, there is no published studies focusing on the metabolic changes between SLE patients with and without skin lesion.

In the present study, we investigated the metabolome of serum samples from SLE patients with or without skin lesion and healthy controls through gas chromatographymass spectrometry (GC-MS). We aimed to identify the disturbed metabolic features and provide novel insights into the pathogenesis of skin lesion in SLE patients, as well as finding potential biomarkers.

\section{Materials and Methods}

\section{Sample Collection}

Twenty-one SLE patients and 16 gender- and age-matched healthy controls (HC) were enrolled in. The patients were recruited from Shanghai General hospital from January 2020 to December 2020 and all of them are Chinese Han population. The inclusion criteria included: (1) patients diagnosed by the 1997 American College of Rheumatology (ACR) revised criteria ${ }^{12}$ for classification of SLE; (2) patients and healthy controls avoided high-fat and high-protein diet before sampling which aimed to eliminate the influence of different diets on metabolomic. The exclusion criteria included: (1) patients with infections or malignancies; (2) patients and healthy controls received systemic antibiotics treatment in the 1 month before enrollment. Of 21 patients, six patients were newly diagnosed and without any treatment whereas 15 patients were diagnosed before and treated with different medications. These 15 patients could be divided into three groups according to different treatment options. Disease activity was estimated by the SLE disease activity index (SLEDAI), while active disease was defined as an SLEDAI score $\geq 5$. Smoking is an important driver of cutaneous activity in SLE and would influence the metabolome profile significantly. ${ }^{13}$ In the present study, we confirmed that no patient smoked in each group. Meanwhile, SLE patients in the present study had no serious comorbidities, such as cardiovascular disease, infections and malignancies which could affect metabolic profile. Erythrocyte sedimentation rate (ESR), complements, $\mathrm{C}$ reactive protein (CRP) and autoantibodies were recorded at the time of sample collection. Fasting blood samples were taken in the morning. Then, serum samples were treated by centrifuging at $2200 \mathrm{rpm}$ for $10 \mathrm{~min}$ and stored at $-80^{\circ} \mathrm{C}$ immediately. This study was approved by the ethics committee of Shanghai General Hospital and all patients signed informed consent. This study was conducted in accordance with the Declaration of Helsinki.

\section{Serum Sample Preparation}

About $20 \mu \mathrm{L}$ mixed internal standards (2-chloro-1-phenylalanine, $0.3 \mathrm{mg} / \mathrm{mL}$ in methanol) were added into $80 \mu \mathrm{L}$ thawed serum sample, shaking for $10 \mathrm{~s}$. Next, $240 \mu \mathrm{L}$ of icecold methanol and acetonitrile $(2: 1, \mathrm{v} / \mathrm{v})$ was added. The sample was vortexed for $30 \mathrm{~s}$ and sonicated by ultrasound for $10 \mathrm{~min}$, and then incubated at $-20{ }^{\circ} \mathrm{C}$ for $30 \mathrm{~min}$. Subsequently, the mixture was centrifuged at $13,000 \mathrm{rpm}$, $4{ }^{\circ} \mathrm{C}$ for $10 \mathrm{~min}$. About $150 \mu \mathrm{L}$ of supernatant was collected and dried under vacuum at room temperature. A two-step procedure was performed to derivatize the residue before GC-MS analysis. First, the sample was redissolved with 80 $\mu \mathrm{L}$ methoxyamine, followed by vortexed and kept at $37^{\circ} \mathrm{C}$ for $90 \mathrm{~min}$. Second, the sample was mixed with $20 \mu \mathrm{L}$ n-hexane and $50 \mu \mathrm{L}$ BSTFA (with 1\% TMCS), vortexed vigorously and derivatized for $1 \mathrm{~h}$ at $70{ }^{\circ} \mathrm{C}$. The quality control samples (QCs) were made by equal amounts of supernatant from each sample. 


\section{Instrumentation and Analytical Conditions}

The pretreated samples were detected by an Agilent 7890B gas chromatography system coupled with an Agilent 5977A MSD system (Agilent Technologies Inc, CA, USA). A DB-5MS fused-silica capillary column (30 $\mathrm{m} \times$ $0.25 \mathrm{~mm} \times 0.25 \mu \mathrm{m}$, Agilent $\mathrm{J} \& \mathrm{~W}$ Scientific, Folsom, CA, USA) was used to separate samples. The processed sample $(1 \mu \mathrm{L})$ was injected in splitless mode, with pure helium as the carrier gas $(1.0 \mathrm{~mL} / \mathrm{min})$. GC was conducted in the temperature programming mode and injector temperature was $260{ }^{\circ} \mathrm{C}$. The parameters of MS were as follows: ion source temperature of $230^{\circ} \mathrm{C}$, MS quadrupole temperature of $150^{\circ} \mathrm{C}$, collision energy of $70 \mathrm{eV}$. A full scan MS data ( $\mathrm{m} / \mathrm{z} 50-500)$ was acquired. The QCs were used every 10 runs to provide repeatable data.

\section{Metabolomics Profiling}

The raw data from GC-MS were processed by the MSDIAL software and Analysis Base File Converter software. Metabolites were identified via LUG database (Lumingbio Untargeted database of GC-MS). A threedimensional matrix was acquired by removing pseudo positive peaks and all internal standards. For multivariate statistical analysis, orthogonal partial least squaresdiscriminant analysis (OPLS-DA), as well as partial least squares-discriminant analysis (PLS-DA) were performed by SIMCA 12.0 software (Umetrics, Sweden). Metabolic pathway analysis was carried out through MetaboAnalyst 4.0, Human Metabolome Database (HMDB) and the Kyoto Encyclopedia of Genes and Genomes (KEGG).

\section{Statistical Analysis}

The clinical features were expressed as median (mean) or median (range). The Shapiro-Wilk test was used to test the normality of the data distribution. Ordinary one-way ANOVA was used to compare the age and BMI of three data sets, while unpaired $t$-test was used for other clinical features. Differential metabolites were defined as the variable importance in the projection (VIP) threshold (VIP > $1.0)$ and two-tailed Student's $t$-test $(p<0.05)$. Receiver operating characteristic (ROC) curve analysis was performed to estimate the diagnostic value of significant differential metabolites. Unpaired $t$-test and MannWhitney test were used to analyze the effect of drugs on metabolites by GraphPad Prism 8.0.

\section{Results}

\section{Clinical Features of Participants}

Twenty-one SLE patients participated in the study were divided into two groups: including patients with skin lesion $(\mathrm{SL}, \mathrm{n}=11)$ and patients without skin lesion $(\mathrm{SNL}, \mathrm{n}=10)$. The median age of SL patients was 40 years, and $91 \%$ of them were female. Meanwhile, the median age of SNL patients was 39.5 years, with $90 \%$ being female. Other manifestations include renal damage, arthralgia, thrombocytopenia, oral ulcers and fever. Moreover, no significant differences in ESR, complements levels, CRP, and autoantibodies were observed between SL and SNL patients, respectively. In 21 SLE patients, 11 patients (52.4\%) were active, and $10(47.6 \%)$ were inactive. In subgroups, the frequency of active patients was $54.5 \%$ in SL group, while that of SNL patients was $50 \%$. The classifications of medication treatments were as follows: untreated $(n=6)$, hydroxychloroquine alone $(\mathrm{n}=4)$, hydroxychloroquine and glucocorticoid $(n=6)$, hydroxychloroquine, glucocorticoid combined with cyclophosphamide $(n=5)$. Clinical features of participants are shown in Table 1. The details of specific drugs, dose of each patient are listed in Table S1.

\section{Serum Metabolome Profiles Can Distinguish Different Groups}

To identify the metabolome features, a total of 37 serum samples from SLE patients $(\mathrm{n}=21)$ and $\mathrm{HC}(\mathrm{n}=16)$ were examined by GC-MS. In PLS-DA model, the SLE patients were clearly distinguished from $\mathrm{HC}$, showing that there were different metabolites between SLE patients and HC (Figure 1). Next, OPLS-DA models were constructed to discriminate different subgroups. The results showed that clear separations between SL and SNL patients (Figure 2A), SL patients and HC (Figure 2B), SNL patients and $\mathrm{HC}$ (Figure 2C) were detected by OPLSDA, respectively. Based on the S-plot of SL and SNL patients, we selected L-alpha-aminobutyric acid and dehydroascorbic acid for potential use of biomarkers in distinguishing these two subgroups (Figure 2D).

\section{Altered-Metabolites in SL Patients, SNL Patients and HC Were Significantly Different}

We further investigated the different distribution of metabolites among SL patients, SNL patients and HC. Features with VIP scores $>1.0$ and $p$ values $<0.05$ were considered as the most significant metabolites. When 
Table I Characteristics of the Study Participants

\begin{tabular}{|c|c|c|c|}
\hline \multirow[t]{2}{*}{ Characteristics } & \multicolumn{2}{|c|}{ SLE } & \multirow[t]{2}{*}{$\mathrm{HC}(\mathrm{N}=16)$} \\
\hline & $S L(N=I I)$ & SNL $(N=10)$ & \\
\hline Gender (M/F) & $1 / 10$ & $1 / 9$ & $2 / 14$ \\
\hline Age (years) ${ }^{a}$ & $40(36.18)$ & $39.5(41.40)$ & $38(33.06)$ \\
\hline $\operatorname{BMl}\left(\mathrm{kg} / \mathrm{m}^{2}\right)^{\mathrm{a}}$ & $22.1(21.53)$ & 22.46 (22.59) & $21.28(20.92)$ \\
\hline \multicolumn{4}{|l|}{ Disease manifestations } \\
\hline Skin lesions ${ }^{c}$ & $1 \mathrm{I} / \mathrm{I}$ (100\%) & $0 / 10(0 \%)$ & - \\
\hline Renal damage $^{c}$ & $4 / I I(36.3 \%)$ & $4 / 10$ (40.0\%) & - \\
\hline Arthralgia ${ }^{c}$ & $2 / 11(18.2 \%)$ & $2 / 10(20.0 \%)$ & - \\
\hline Thrombocytopenia $^{c}$ & I/II (9.0\%) & $1 / 10(10.0 \%)$ & - \\
\hline Oral ulcers ${ }^{c}$ & I/II (9.0\%) & $0 / 10(0 \%)$ & - \\
\hline Fever $^{c}$ & I/II (9.0\%) & $1 / 10(10.0 \%)$ & - \\
\hline \multicolumn{4}{|l|}{ Disease activity parameters } \\
\hline $\operatorname{ESR}(\mathrm{mm} / \mathrm{h})^{a}$ & $10(18.09)$ & $14(20.40)$ & - \\
\hline $\mathrm{CRP}(\mathrm{mg} / \mathrm{L})^{\mathrm{a}}$ & $2(8.56)$ & $2.15(8.61)$ & - \\
\hline $\mathrm{C} 3(\mathrm{~g} / \mathrm{L})^{\mathrm{a}}$ & $0.87(0.84)$ & $0.96(0.86)$ & - \\
\hline $\mathrm{C} 4(\mathrm{~g} / \mathrm{L})^{\mathrm{a}}$ & $0.16(0.15)$ & $0.15(0.15)$ & - \\
\hline SLEDAI ${ }^{b}$ & $6(2,16)$ & $4(2,15)$ & - \\
\hline -Active patients ${ }^{c}$ & $6 / 11(54.5 \%)$ & $5 / 10(50 \%)$ & - \\
\hline -Inactive patients ${ }^{c}$ & $5 / 11(45.5 \%)$ & $5 / 10(50 \%)$ & - \\
\hline \multicolumn{4}{|l|}{ Autoantibody status } \\
\hline Anti-dsDNA $\lg G(I U / m L){ }^{a}$ & $402.5(475.26)$ & $346.0(376.89)$ & - \\
\hline Positive anti-ds DNA ${ }^{c}$ & $6 / 11$ (54.5\%) & $5 / 10(50 \%)$ & - \\
\hline Positive ANA ${ }^{c}$ & $9 / 11$ (81.2\%) & $8 / 10(80.0 \%)$ & - \\
\hline \multicolumn{4}{|l|}{ Treatment options } \\
\hline Untreated $^{c}$ & \multicolumn{2}{|c|}{$6 / 21(28.6 \%)$} & - \\
\hline $\mathrm{HCQ}^{\mathrm{c}}$ & \multicolumn{2}{|c|}{$4 / 21$ (19.0\%) } & - \\
\hline $\mathrm{HCQ}+\mathrm{G}^{\mathrm{c}}$ & \multicolumn{2}{|c|}{$6 / 21(28.6 \%)$} & - \\
\hline $\mathrm{HCQ}+\mathrm{G}+\mathrm{CYC}^{\mathrm{c}}$ & \multicolumn{2}{|c|}{$5 / 21$ (23.8\%) } & - \\
\hline
\end{tabular}

Notes: ${ }^{a}$ Median (mean); ${ }^{\mathrm{b}}$ median (range); ${ }^{\mathrm{c}} \mathrm{n} / \mathrm{N}$ (percentage).

Abbreviations: M, male; F, female; BMI, body mass index; ESR, erythrocyte sedimentation rate; CRP, C reactive protein; C3, complement 3; C4, complement 4; SLEDAI, systemic lupus erythematosus disease activity index; HCQ, Hydroxychloroquine; G, Glucocorticoid; CYC, Cyclophosphamide.

comparing SL patients with SNL patients, a total of 14 differential metabolites were found (Table 2). Levels of L-alpha-aminobutyric acid, acrylic acid, glycine, L-aspartic, indolelactic acid, L-tyrosine, nicotinic acid, glucosamine, O-phosphoethanolamine, and rhamnose were increased in SL group. In addition, levels of dehydroascorbic acid, gluconic acid lactone, and putrescine were decreased in SL group. Moreover, beta-alanine and L-threonine were decreased in both SL and SNL patients compared to HC. 


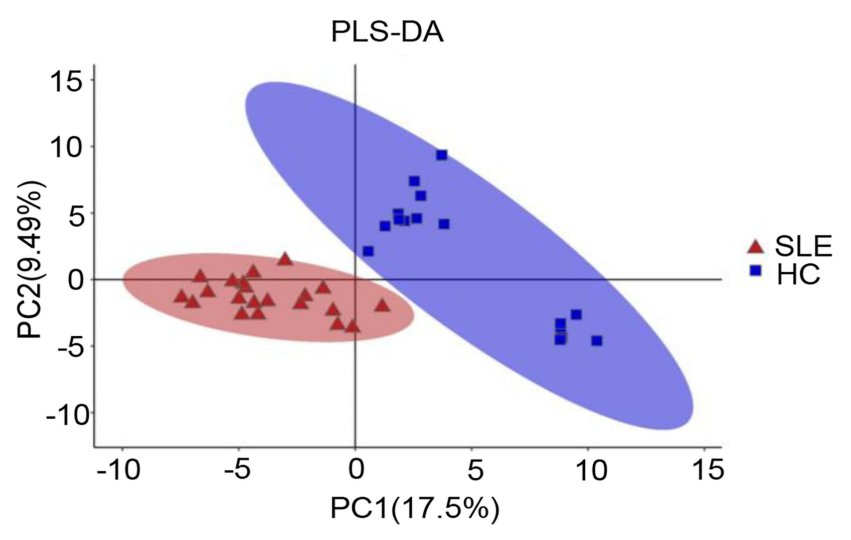

Figure I PLS-DA of serum metabolomics data distinguished total SLE patients $(n=$ 21) from HC $(n=16)$. The red triangles represented total SLE patients while the blue squares represented $\mathrm{HC}$ in the PLS-DA score plots.

Abbreviations: PLS-DA, partial least squares discriminant analysis; SLE, systemic lupus erythematosus; $\mathrm{HC}$, healthy controls.

\section{Metabolic Pathway Analysis}

To identify the distinguished pathways in SL patients, analysis was performed through KEGG metabolic library. Nineteen significant pathways with $p$ values $<0.05$ were detected (Figure 3A). Glutathione metabolism $(p=0.0003)$ was the most altered pathway in SL patients. The other top nine metabolic pathways included four amino-related pathways: aminoacyl-tRNA biosynthesis, protein digestion and absorption, glycine, serine and threonine metabolism, cysteine and methionine metabolism, and five other important pathways: central carbon metabolism in cancer, thiamine metabolism, neuroactive ligand-receptor interaction, nicotinate and nicotinamide metabolism, melanogenesis.

The decreased dehydroascorbic acid and putrescine, and increased glycine were involved in glutathione metabolism. Meanwhile, increased glycine, L-aspartic acid, L-tyrosine were enriched in aminoacyl-tRNA biosynthesis.

Considering the metabolomics might be different between active ( $\mathrm{SLEDAI} \geq 5$ ) and inactive patients (SLEDAI $\leq 4)$, we carried out further analysis. The result of OPLS-DA (Figure S1) showed that metabolomics could distinguish active patients from inactive patients. Meanwhile, several significant pathways, such as glycine, serine and threonine metabolism, cysteine and methionine pathway, as well as taurine and hypotaurine metabolism were enriched (Figure 3B).

\section{Several Serum Metabolites Showed Diagnostic Potential for SLE Patients with Skin Involvement}

To reveal the differential capacity of metabolites in distinguishing SL patients from SNL patients, ROC curve
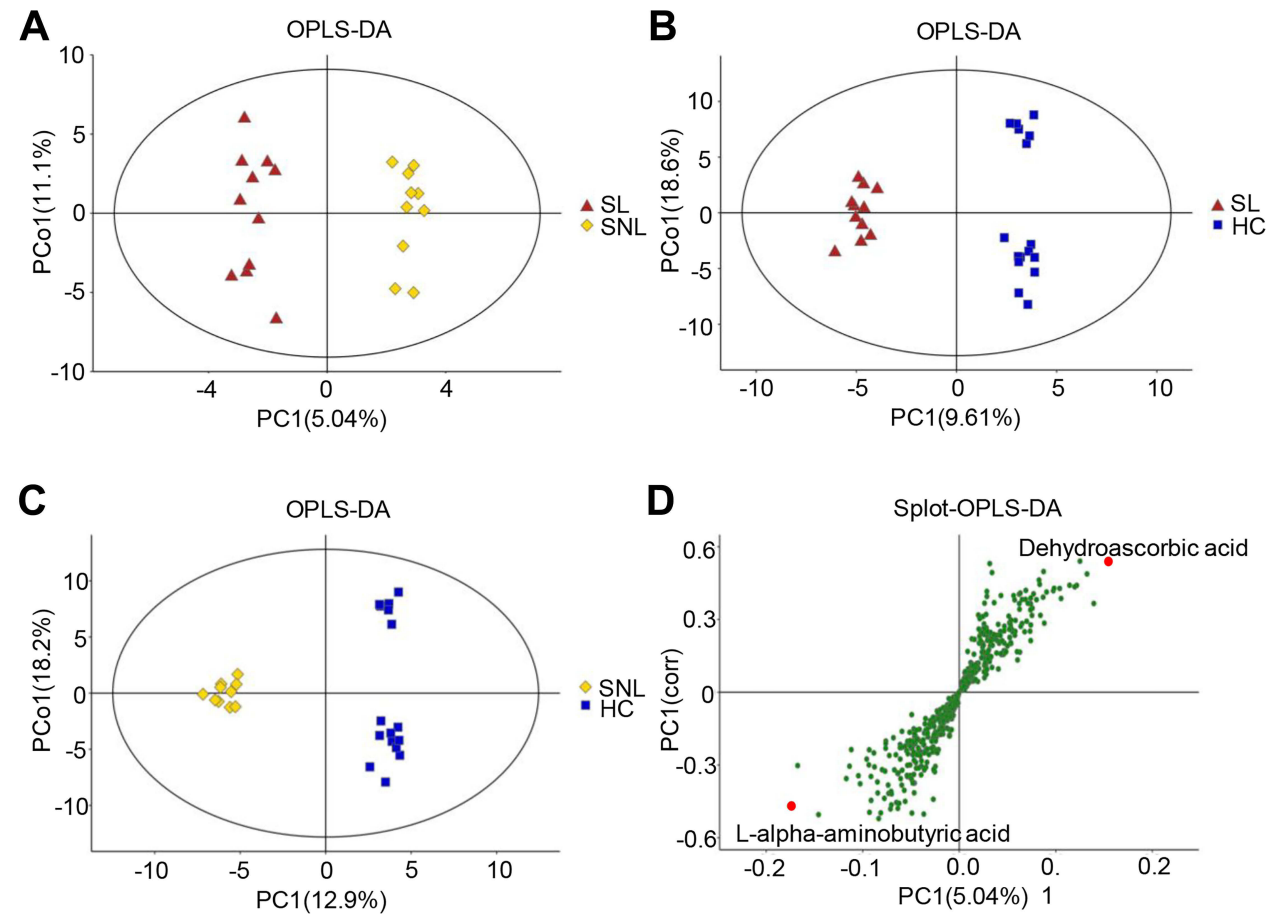

Figure 2 OPLS-DA could distinguish different subgroups and S-plot helps to select the potential biomarkers. (A) SL patients (red triangles) compared with SNL patients (yellow rhombus); (B) SL patients (red triangles) compared with HC (blue squares); (C) SNL patients (yellow rhombus) compared with HC (blue squares); (D) L-alphaaminobutyric acid and dehydroascorbic acid were considered as potential biomarkers based on the S-plot.

Abbreviations: OPLS-DA, orthogonal projection latent to structure discriminant analysis; SL patients, SLE patients with skin lesions; SNL, SLE patients without skin lesions. 
Table 2 Serum Identified Differential Metabolites Between Groups

\begin{tabular}{|c|c|c|c|c|c|c|c|c|c|}
\hline \multirow[t]{2}{*}{ Metabolites } & \multicolumn{3}{|c|}{ SL vs SNL } & \multicolumn{3}{|c|}{ SL vs HC } & \multicolumn{3}{|c|}{ SNL vs HC } \\
\hline & $\mathbf{v}$ & VIP & FC & $\mathbf{v}$ & VIP & FC & $\mathbf{v}$ & VIP & FC \\
\hline L-alpha-aminobutyric acid & $\uparrow$ & 3.91 & 3.68 & - & - & - & - & - & - \\
\hline Dehydroascorbic acid & $\downarrow$ & 2.78 & 0.39 & - & - & - & - & - & - \\
\hline Gluconic acid lactone & $\downarrow$ & 2.32 & 0.43 & $\uparrow$ & 2.13 & 3.06 & $\uparrow$ & 2.60 & 7.11 \\
\hline Putrescine & $\downarrow$ & 2.17 & 0.16 & - & - & - & - & - & - \\
\hline Acrylic acid & $\uparrow$ & 1.97 & 1.78 & - & - & - & - & - & - \\
\hline Delta-tocopherol & $\downarrow$ & 1.94 & 0.66 & $\downarrow$ & 1.12 & 0.53 & - & - & - \\
\hline Glycine & $\uparrow$ & 1.85 & 1.50 & - & - & - & - & - & - \\
\hline L-aspartic acid & $\uparrow$ & 1.81 & 1.52 & - & - & - & - & - & - \\
\hline Indolelactic acid & $\uparrow$ & 1.59 & 1.45 & - & - & - & - & - & - \\
\hline L-tyrosine & $\uparrow$ & 1.57 & 1.41 & - & - & - & $\downarrow$ & 1.21 & 0.63 \\
\hline Nicotinic acid & $\uparrow$ & 1.45 & 1.38 & - & - & - & - & - & - \\
\hline Glucosamine & $\uparrow$ & 1.42 & 1.28 & - & - & - & $\downarrow$ & 1.09 & 0.69 \\
\hline O-phosphoethanolamine & $\uparrow$ & 1.39 & 1.33 & - & - & - & - & - & - \\
\hline Rhamnose & $\uparrow$ & 1.10 & 1.19 & $\uparrow$ & 1.86 & 1.57 & - & - & - \\
\hline Beta-alanine & - & - & - & $\downarrow$ & 1.62 & 0.47 & $\downarrow$ & 1.62 & 0.41 \\
\hline L-glutamine & - & - & - & $\uparrow$ & 2.42 & 3.39 & $\uparrow$ & 1.68 & 2.79 \\
\hline L-threonine & - & - & - & $\downarrow$ & 1.01 & 0.74 & $\downarrow$ & 1.20 & 0.63 \\
\hline
\end{tabular}

Notes: $\uparrow$ Represents upregulation of metabolite; $\downarrow$ represents downregulation of metabolite. Abbreviations: V, variation; VIP, variable importance in the projection; FC, fold change.

analysis was performed. In addition to L-alphaaminobutyric acid and dehydroascorbic acid, the other top eight significant metabolites ranked by VIP scores between the SL and SNL group were also analyzed. The results demonstrated that an area under the ROC curve (AUC) of four metabolites, L-alpha-aminobutyric acid $(\mathrm{AUC}=0.8636)$, dehydroascorbic acid $(\mathrm{AUC}=0.8091)$, glycine $(\mathrm{AUC}=0.7727)$, and L-tyrosine $(\mathrm{AUC}=0.7636)$, respectively, indicating their good differential performance (Figure 4A-D). Furthermore, to enhance the differential capacity for SL patients, a combined model was established. The combination of L-alpha-aminobutyric acid and dehydroascorbic acid achieved an AUC of 0.9273 , with specificity of 0.9 and sensitivity of 0.82 , showing increased predictive capacity (Figure 4E).

\section{Effects of Medications on Metabolites}

Drugs usually have huge impacts on the metabolite profile, and some of our patients were under different medication treatment when collected serum samples (Table 1).
A

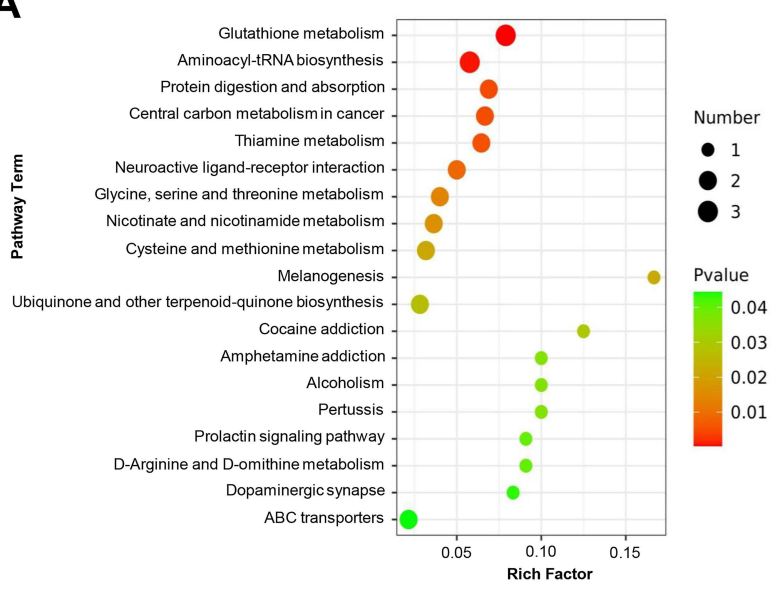

B

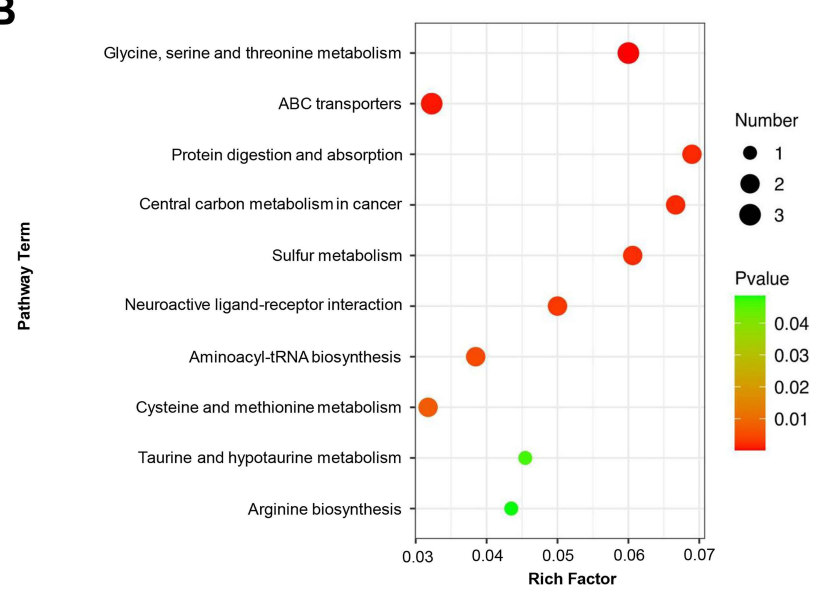

Figure 3 Pathway enrichment of altered metabolites. (A) Metabolites pathway enrichment between SL and SNL patients showed glutathione metabolism and aminoacyltRNA biosynthesis were significantly disturbed $(p<0.0$ I). (B) Pathway enrichment of altered metabolites between active and inactive patients. Circle colors represented pathway enrichment significance. Circle size indicated the number of metabolites in the specific pathway. The $X$-axis represented the rich factor, while the $Y$-axis represented pathways. 

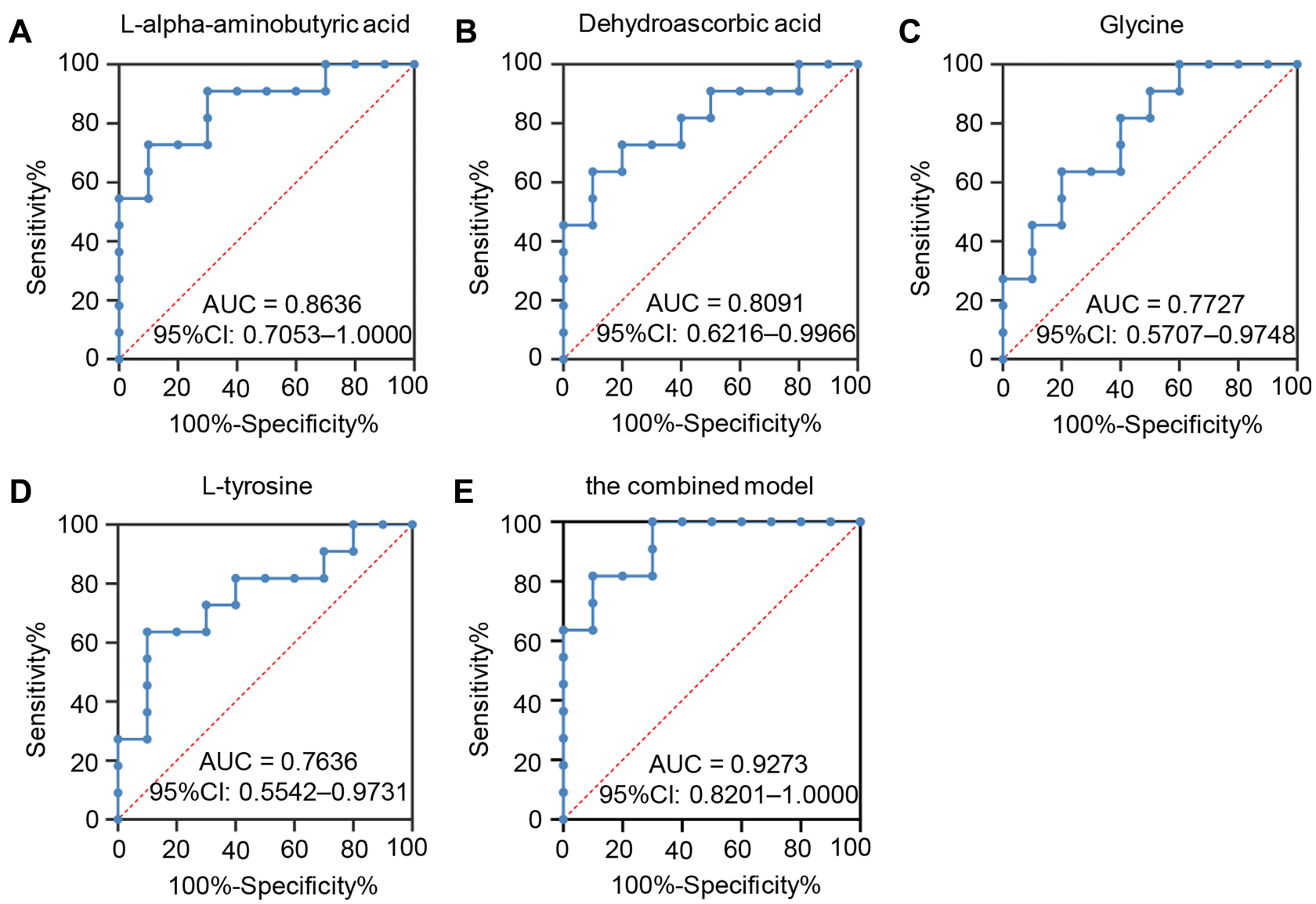

Figure 4 ROC analysis of potential biomarkers for differentiating SLE patients with or without rashes. (A) L-alpha-aminobutyric acid showed an AUC of 0.8636 ( $95 \%$ Cl: $0.7053-1.000, p=0.0049)$. (B) Dehydroascorbic acid presented an AUC of 0.809I (95\% Cl: 0.6216-0.9966, $p=0.0167)$. (C) Glycine revealed an AUC of 0.7727 (95\% Cl: $0.5707-0.9748, p=0.0346)$ and the AUC value of L-tyrosine $(D)$ was $0.7636(95 \% \mathrm{Cl}: 0.5542-0.973 \mathrm{I}, p=0.04 \mathrm{I}$ ). (E) The combined model of L-alpha-aminobutyric acid and dehydroascorbic acid achieved an AUC of 0.9273.

Abbreviation: ROC, receiver operating characteristic.

Therefore, we examined whether L-alpha-aminobutyric acid and dehydroascorbic acid were influenced by medications. Different medication groups were compared to each other. The results showed that hydroxychloroquine, glucocorticoid, as well as cyclophosphamide had no effects on L-alpha-aminobutyric acid (Figure 5A-C). Likewise, dehydroascorbic acid (Figure 5D-F) was also not influenced by these medications.

\section{Discussion}

As a rapidly emerging science, metabolomes have been attracting the attention of scientific studies because of high resolution and sensitivity of spectrometry. Other metabolomics studies in SLE have revealed discrimination between SLE and normal controls using the samples of urine, fecal or blood..$^{8-11,14}$ In the current study, we demonstrated that serum metabolome profiles based on GC/MS could distinguish SLE patients with skin lesion from those without lesion and healthy controls. We demonstrated that fourteen metabolites were markedly altered in SL patients compared to SNL patients. Meanwhile, a total of nineteen metabolism pathways were perturbed. Furthermore, we identified that the combination of L-alpha-aminobutyric acid and dehydroascorbic acid might predict the development of skin involvement in SLE patients. To our knowledge, this is the first study focused on changes of metabolomics in SLE patients with skin involvement.

According to published studies, L-threonine, a kind of amino acid, was reduced in SLE plasma compared with HCs. ${ }^{15}$ Moreover, Zhou et $\mathrm{al}^{14}$ revealed that SLE sera samples were characterized by decreased Betaalanine using ${ }^{1} \mathrm{H}$ NMR spectroscopy-based metabolomics. Consistently with their results, the reduced serum L-threonine and Beta-alanine were detected in our study indicating our results were solid and stable. 

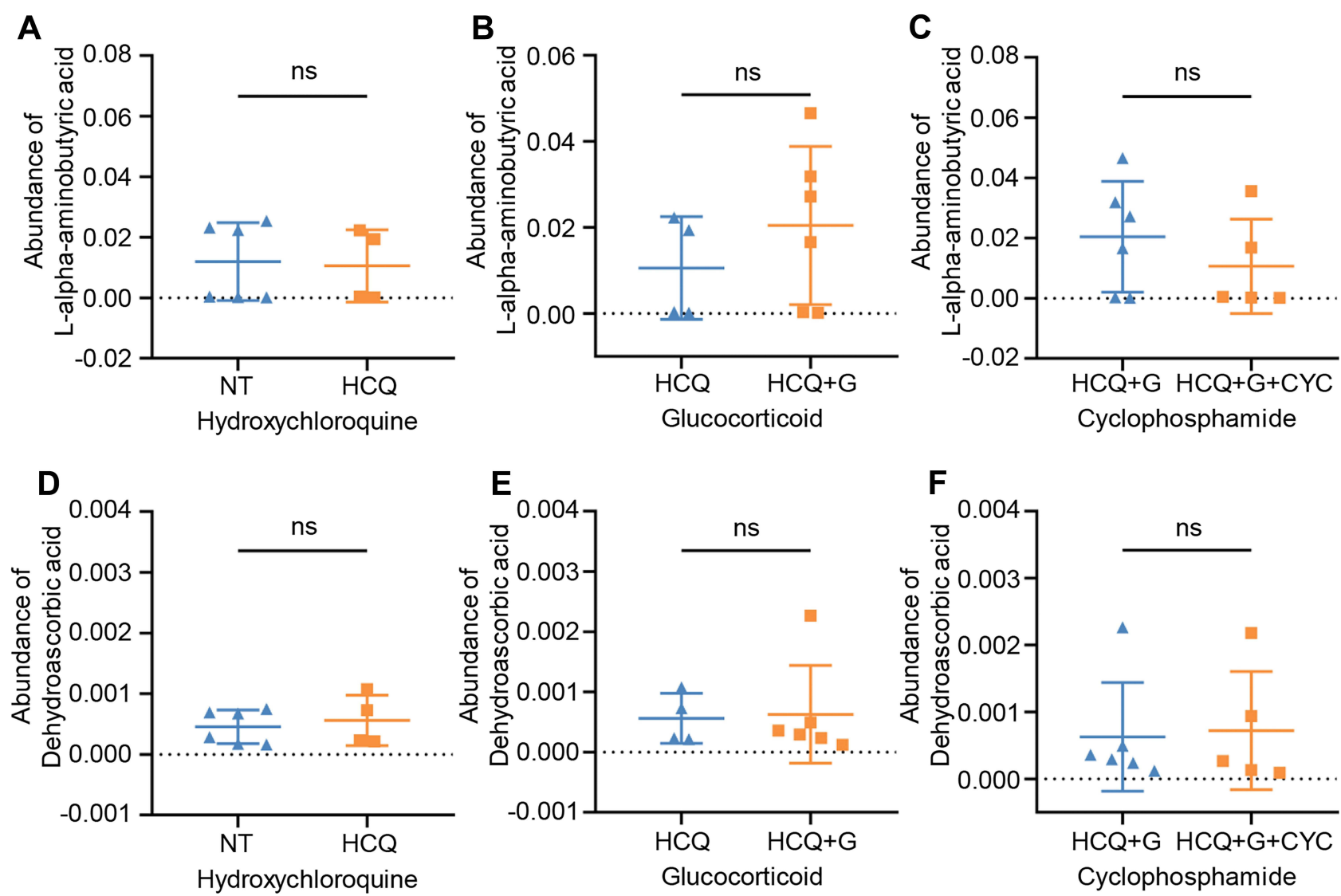

Figure 5 The effect of different medications on L-alpha-aminobutyric acid and dehydroascorbic acid. Treatment of hydroxychloroquine (A and D), glucocorticoid (B and E) and cyclophosphamide ( $\mathbf{C}$ and $\mathbf{F}$ ) did not provoke considerable variation of L-alpha-aminobutyric acid and dehydroascorbic acid. Statistical significance was performed by Mann-Whitney test and unpaired $t$-test.

Abbreviations: ns, not significant; NT, not treated with specific drugs; HCQ, hydroxychloroquine; G, glucocorticoid; CYC, cyclophosphamide.

Dehydroascorbic acid (DHA, VIP $=2.78, \mathrm{FC}=0.39$ ) is the oxidized form of vitamin C. ${ }^{16}$ Previous study indicated that vitamin $\mathrm{C}$ was involved in immune response by modulating cytokine secretion and $\mathrm{T}$ cell proliferation. Moreover, Th1-shifting effects of vitamin $\mathrm{C}$ had also been observed. ${ }^{17-20} \mathrm{~T}$ cells intake vitamin $\mathrm{C}$ mostly in its oxidized form, DHA. ${ }^{19}$ Our data demonstrated that the level of DHA in SL patients was lower than SNL patients, indicating that $\mathrm{T}$ cells of SL patients might intake more serum DHA and maybe shift into Th1. According to previous studies, Th1 might contribute to the development of SLE skin inflammation. ${ }^{21}$ Therefore, the regulatory mechanism of DHA on immune needs further investigation, which may be useful to research new therapeutic targets for SLE patients with skin injury.

Another significant altered metabolite was L-alphaaminobutyric acid (VIP $=3.91, \mathrm{FC}=3.68)$, which was involved in cysteine and methionine metabolism, and we showed it was unregulated in SL patients compared to SNL patients. The abnormal cysteine and methionine metabolism was also observed in vitiligo patients compared to $\mathrm{HC}$ by Liu et al. ${ }^{22}$ It was reported that aberrant homocysteine metabolism may lead to increased oxidative damage, excessive IL-6 production, and then trigger autoimmunity and the development of vitiligo. ${ }^{23,24}$ Likewise, excessive oxidative damage was also a characteristic of SLE. ${ }^{25}$ Meanwhile, high IL-6 expression was identified in skin lesion of SLE patients, ${ }^{26}$ and the increased IL-6 expression might amplify the inflammation in lupus. ${ }^{27}$ These findings implied that the enriched cysteine and methionine metabolism pathways found in our study might contribute to the skin lesion in SLE patients via oxidative damage and IL- 6 production.

The glutathione metabolism pathway was the most significant perturbed pathway in SL patients in our study, indicating skin injury in SLE was related with oxidative damage furtherly. Consistently, a previous $\operatorname{study}^{28}$ has identified the dysregulated glutathione metabolism in SLE. Yan et $\mathrm{al}^{15}$ also showed that glutamate was related to SLE skin injury, providing the evidence of oxidant/ 
antioxidant imbalance in the pathogenesis of SLE skin involvement.

Furthermore, nicotinamide metabolism pathway which is related with DNA repair was enriched in this study. Previous evidence demonstrated that oxidized DNA damage was increased in skin lesion of SLE patients, due to the exposure of ultraviolet light and the less expression of oxoguanine glycosylase 1 (OGG1). ${ }^{29}$ Thereby, this enriched pathway may be results of cumulative DNA damage in skin, suggesting that organ damage may lead to the changes of overall metabolism. Glycine, serine and threonine metabolism and cysteine and methionine pathway were detected when active and inactive patients were compared, suggesting these pathways might be involved in the development of SLE and related with the disease activity. Meanwhile, $\mathrm{Li}$ et $\mathrm{al}^{30}$ revealed that taurine metabolism could enhance pDC-mediated type I IFN production and was involved in the development of SLE. We also found that taurine and hypotaurine metabolism pathway was enriched implying this pathway might be correlated with disease activity.

Individual and combined metabolites could be potential good biomarkers for different diseases. It was reported that the AUC value of combination of 2,2-dimethylsuccinic acid, beta-alanine and DOPAL was 0.89 , exhibited good predictive ability of SLE. ${ }^{11}$ Similarly, we found that the AUC values of individual L-alpha-aminobutyric acid, dehydroascorbic acid, were $0.8636,0.8091$, respectively. Furthermore, the combination of L-alpha-aminobutyric acid and dehydroascorbic acid $(\mathrm{AUC}=0.9273)$ predicted the skin involvement in SLE patients better.

Metabolites could be influenced by different medications. Previous studies had revealed that hydroxychloroquine could affect lipid metabolism in SLE patients. ${ }^{31,32}$ However, Zhang et $\mathrm{al}^{8}$ found hydroxychloroquine and glucocorticoid were not correlated with serum metabolite, L-pyroglutamic. Consistently, in our study, hydroxychloroquine, glucocorticoid and cyclophosphamide had no significant effect on L-alpha-aminobutyric acid and dehydroascorbic acid, indicating these metabolites were stable biomarkers.

There are some limitations in this study. Firstly, the present study was relatively limited by small sample sizes and studies with larger number of samples are required. Moreover, in order to validate clinical utility and specificity of metabolites as biomarkers for SLE patients with skin lesion, additional control groups with other inflammatory or autoimmune diseases also need to be considered. In this study, we identified differential metabolites and metabolites-related pathways in SLE patients with or without skin lesion. Meanwhile, a panel of four candidate biomarkers was proposed and a combined model might achieve a good predictive performance for SLE patients with skin lesion. These results open a new avenue to investigate the mechanism of SLE skin involvement, and might provide potential targets for treating skin lesion and prevent systemic flare.

\section{Funding}

This work was supported by NSFCGrant (Grant Number, 81773310 and 81402587).

\section{Disclosure}

The authors have declared that there is no conflict of interest.

\section{References}

1. Tsokos GC. Systemic lupus erythematosus. $N$ Engl J Med. 2011;365 (22):2110-2121. doi:10.1056/NEJMra1100359

2. Deng GM, Tsokos GC. Pathogenesis and targeted treatment of skin injury in SLE. Nat Rev Rheumatol. 2015;11:663-669. doi:10.1038/ nrrheum.2015.106

3. Yell JA, Mbuagbaw J, Burge SM. Cutaneous manifestations of systemic lupus erythematosus. Br J Dermatol. 1996;135(3):355-362. doi:10.1046/j.1365-2133.1996.d01-1004.x

4. Kuhn A, Wenzel J, Weyd H. Photosensitivity, apoptosis, and cytokines in the pathogenesis of lupus erythematosus: a critical review. Clin Rev Allergy Immunol. 2014;47(2):148-162. doi:10.1007/s12016013-8403-x

5. Xie Y, Liu B, Wu Z. Increased interleukin-9 levels in skin lesion from cutaneous lupus erythematosus patients may predict the progression to systemic lupus erythematosus. J Dermatol Sci. 2021;101:78-80. doi:10.1016/j.jdermsci.2020.10.016

6. Deng GM, Liu L, Kyttaris VC, Tsokos GC. Lupus serum IgG induces skin inflammation through the TNFR1 signaling pathway. J Immunol. 2010;184:7154-7161. doi:10.4049/jimmunol.0902514

7. Yu H, Jiang L, Liu R, et al. Association between the ratio of aryl hydrocarbon receptor (AhR) in Th17 cells to AhR in treg cells and SLE skin lesions. Int Immunopharmacol. 2019;69:257-262. doi:10.1016/j.intimp.2019.01.039

8. Zhang Q, Li X, Yin X, et al. Metabolomic profiling reveals serum L-pyroglutamic acid as a potential diagnostic biomarker for systemic lupus erythematosus. Rheumatology. 2021;60:598-606. doi:10.1093/ rheumatology/keaa126

9. Yan R, Jiang H, Gu S, et al. Fecal metabolites were altered, identified as biomarkers and correlated with disease activity in patients with systemic lupus erythematosus in a GC-MS-based metabolomics study. Front Immunol. 2020;11:2138. doi:10.3389/fimmu.2020.02138

10. Zhang Q, Yin X, Wang H, et al. Fecal metabolomics and potential biomarkers for systemic lupus erythematosus. Front Immunol. 2019;10:976. doi:10.3389/fimmu.2019.00976

11. Kalantari S, Chashmniam S, Nafar M, Zakeri Z, Parvin M. Metabolomics approach reveals urine biomarkers and pathways associated with the pathogenesis of lupus nephritis. Iran J Basic Med Sci. 2019;22:1288-1295. doi:10.22038/ijbms.2019.38713.9178 
12. Hochberg MC. Updating the American college of rheumatology revised criteria for the classification of systemic lupus erythematosus. Arthritis Rheum. 1997;40(9):1725. doi:10.1002/ art. 1780400928

13. Hsu P-C, Lan RS, Brasky TM. Metabolomic profiles of current cigarette smokers. Mol Carcinog. 2017;56(2):594-606. doi:10.1002/ mc.22519

14. Zhou YJ, Li LS, Sun JL, Guan K, Wei JF. (1)H NMR-based metabolomic study of metabolic profiling for pollinosis. World Allergy Organ J. 2019;12:100005. doi:10.1016/j.waojou.2018.11.005

15. Yan B, Huang J, Zhang C, et al. Serum metabolomic profiling in patients with systemic lupus erythematosus by GC/MS. Mod Rheumatol. 2016;26:914-922. doi:10.3109/14397595.2016.1158895

16. Baldermann S, Homann T, Neugart S, et al. Selected plant metabolites involved in oxidation-reduction processes during bud dormancy and ontogenetic development in sweet cherry buds (Prunus avium L.). Molecules. 2018;23(5):1197. doi:10.3390/molecules23051197

17. Jeong YJ, Hong SW, Kim JH, et al. Vitamin C-treated murine bone marrow-derived dendritic cells preferentially drive naïve $\mathrm{T}$ cells into Th1 cells by increased IL-12 secretions. Cell Immunol. 2011;266:192-199. doi:10.1016/j.cellimm.2010.10.005

18. Kennes B, Dumont I, Brohee D, Hubert C, Neve P. Effect of vitamin $\mathrm{C}$ supplements on cell-mediated immunity in old people. Gerontology. 1983;29:305-310. doi:10.1159/000213131

19. Maeng HG, Lim H, Jeong YJ, et al. Vitamin C enters mouse T cells as dehydroascorbic acid in vitro and does not recapitulate in vivo vitamin C effects. Immunobiology. 2009;214:311-320. doi:10.1016/j. imbio.2008.09.003

20. Wintergerst ES, Maggini S, Hornig DH. Immune-enhancing role of vitamin $\mathrm{C}$ and zinc and effect on clinical conditions. Ann Nutr Metab. 2006;50:85-94. doi:10.1159/000090495

21. Carneiro JR, Fuzii HT, Kayser C, et al. IL-2, IL-5, TNF- $\alpha$ and IFN- $\gamma$ mRNA expression in epidermal keratinocytes of systemic lupus erythematosus skin lesion. Clinics. 2011;66:77-82. doi:10.1590/ s1807-59322011000100014

22. Liu W, Liu XY, Qian YT, et al. Urinary metabolomic investigations in vitiligo patients. Sci Rep. 2020;10:17989. doi:10.1038/s41598-02075135-0
23. Silverberg JI, Silverberg NB. Serum homocysteine as a biomarker of vitiligo vulgaris severity: a pilot study. $J$ Am Acad Dermatol. 2011;64:445-447. doi:10.1016/j.jaad.2010.08.025

24. Tsai TY, Kuo CY, Huang YC. Serum homocysteine, folate, and vitamin B (12) levels in patients with vitiligo and their potential roles as disease activity biomarkers: a systematic review and meta-analysis. J Am Acad Dermatol. 2019;80:646-654.e645. doi:10.1016/j.jaad.2018.08.029

25. Lightfoot YL, Blanco LP, Kaplan MJ. Metabolic abnormalities and oxidative stress in lupus. Curr Opin Rheumatol. 2017;29:442-449. doi: $10.1097 /$ bor.0000000000000413

26. Pflegerl P, Vesely P, Hantusch B, et al. Epidermal loss of JunB leads to a SLE phenotype due to hyper IL-6 signaling. Proc Natl Acad Sci U S A. 2009;106(48):20423-20428. doi:10.1073/pnas.0910371106

27. Robinson ES, Werth VP. The role of cytokines in the pathogenesis of cutaneous lupus erythematosus. Cytokine. 2015;73:326-334. doi:10.1016/j.cyto.2015.01.031

28. Gergely P Jr, Grossman C, Niland B, et al. Mitochondrial hyperpolarization and ATP depletion in patients with systemic lupus erythematosus. Arthritis Rheum. 2002;46(1):175-190. doi:10.1002/ 1529-0131(200201)46:1<175::AID-ART10015>3.0.CO;2-H

29. Tumurkhuu G, Chen S, Montano EN, et al. Oxidative DNA damage accelerates skin inflammation in pristane-induced lupus model. Front Immunol. 2020;11:554725. doi:10.3389/fimmu.2020.554725

30. Li J, Ding H, Meng Y. Taurine metabolism aggravates the progression of lupus by promoting the function of plasmacytoid dendritic cells. Arthritis Rheumatol. 2020;72(12):2106-2117. doi:10.1002/ art.41419

31. Hage MP, Al-Badri MR, Azar ST. A favorable effect of hydroxychloroquine on glucose and lipid metabolism beyond its anti-inflammatory role. Ther Adv Endocrinol Metab. 2014;5 (4):77-85. doi:10.1177/2042018814547204

32. Kavanaugh A, Adams-Huet B, Jain R, Denke M, McFarlin J. Hydroxychloroquine effects on lipoprotein profiles (the HELP trial): a double-blind, randomized, placebo-controlled, Pilot Study in patients with systemic lupus erythematosus. J Clin Rheumatol. 1997;3(1):3-8. doi:10.1097/00124743-199702000-00002

\section{Publish your work in this journal}

Clinical, Cosmetic and Investigational Dermatology is an international, peer-reviewed, open access, online journal that focuses on the latest clinical and experimental research in all aspects of skin disease and cosmetic interventions. This journal is indexed on CAS.
The manuscript management system is completely online and includes a very quick and fair peer-review system, which is all easy to use. Visit http://www.dovepress.com/testimonials.php to read real quotes from published authors. 